\title{
Backlash Algorithm: A trading strategy based on directional change
}

\author{
Amer Bakhach, Edward P. K. Tsang \\ Centre of Computational Finance \\ and Economic Agent \\ University of Essex \\ Colchester, UK \\ abakhaa, edward (@essex.ac.uk)
}

\author{
Wing Lon $\mathrm{Ng}$ \\ www.bracil.net \\ London, UK \\ wlng@bracil.net
}

\author{
V L Raju Chinthalapati \\ Dept. Accounting and Finance \\ University of Greenwich \\ Greenwich, UK \\ v.1.r.chinthalapati@gre.ac.uk
}

\begin{abstract}
Directional Change (DC) is a new way to summarize price movements in a financial market. Unlike time series, it samples data at irregular time intervals. According to the DC concept, the data is sampled only when the magnitude of price changes is significant according to the investor. In this paper, we propose a contrarian trading strategy which is based on the DC concept. We test our trading strategy using two currency pairs; namely EUR/CHF and EUR/USD. The results show that our proposed trading strategy is consistently profitable; it produce a profit of up to $145 \%$ within seven months; whereas the buy-andhold approach incurred a loss of $-14 \%$ during the same trading period.
\end{abstract}

Index Terms - contrarian trading strategy; directional change; FX trading.

\section{INTRODUCTION}

The literature on trading strategies encompasses plenty of trading models. Some of the existing trading strategies were based on forecasting market prices" "turning points" (e.g. [1]) or "directional-of-changes" (e.g. [2]). Other trading models were based on the stylized facts of a specific market (e.g. [3]). Technical trading rules were also used to develop trading strategies (e.g. [4] [5]). The literature also includes trading strategies that embrace momentum models (e.g. [6] [7]). Other researches tried to combine multiple trading strategies (e.g. [8]). The common criteria among all these researches is that the authors developed and tested their trading strategies using time series data. In other words, they sampled market prices at fixed time intervals, let it be days, minutes, etc.

Directional change (DC) is another approach to summarize the movement of financial markets' prices [9]. Under the DC framework, in contrast to time series, the focus is on the magnitude of price change and time is the varying factor [10].

The DC concept had been proved many times to be helpful in the study of the FX market. For instance, [11] reported 12 scaling laws by analyzing 14 different currency pairs using the DC concept. In addition, [12] introduced the so-called Scale of Market Quakes (SMQ) based on the DC concept. SMQ aimed at quantifying the FX market activity at main economic and political events declarations. Furthermore, [13] used the DC concept to present a model that explains how minor differences in the FX market activities can change the price trend under definite conditions.

The results reported in these studies are interesting. However, only few researches tried to develop trading models based on the DC concept. For instance, [14] presented a DCbased contrarian trading strategy; which attempted to exploit the scaling laws in FX market. More recently, [15] introduced a trading strategy which was based on forecasting the daily closing price of a financial market. Their forecasting model embedded a combination of the DC framework and Genetic Programming.

We believe that the usefulness of using the DC concept to develop a trading model is not fully exploited yet. In this paper we provide evidences that the DC concept can be very helpful as the basis of a trading strategy. We introduce a new contrarian trading strategy, named Backlash Algorithm, which is based on the DC concept. We verify that our strategy is consistently profitable. We provide a set of experiments to examine the profitability and in-depth analysis of our proposed strategy. These experiments are conducted using two currency pairs: EUR/CHF and EUR/USD.

This paper continues as follow: Section II provides an overview of the DC concept. Section III describes two types of our trading strategy with the corresponding trading rules. Section IV provides detailed description of our experiments and the evaluation metrics. We report and discuss the results in Section V. We conclude in Section VI.

\section{DIRECTIONAL CHANGE: AN OVERVIEW}

The DC approach focuses on significant changes in price movements. Here, the significance is defined as price changes larger than, or equal to, a predetermined threshold which is the choice of the investor. Let $\theta$ be this threshold. Usually, $\theta$ is expressed in percentage. According to the DC concept, the market can be in downtrend or in uptrend. If we observe a price rise of magnitude $\theta$, we say that the market is in uptrend [10]. Similarly, if we detect a price decline of magnitude $\theta$, we say that the market is in downtrend. An uptrend is directly followed by a downtrend; and vice versa (see Fig.1). The price at which a downtrend, or an uptrend, begins is called $\mathrm{P}_{\text {EXT. }}$ In case of an 


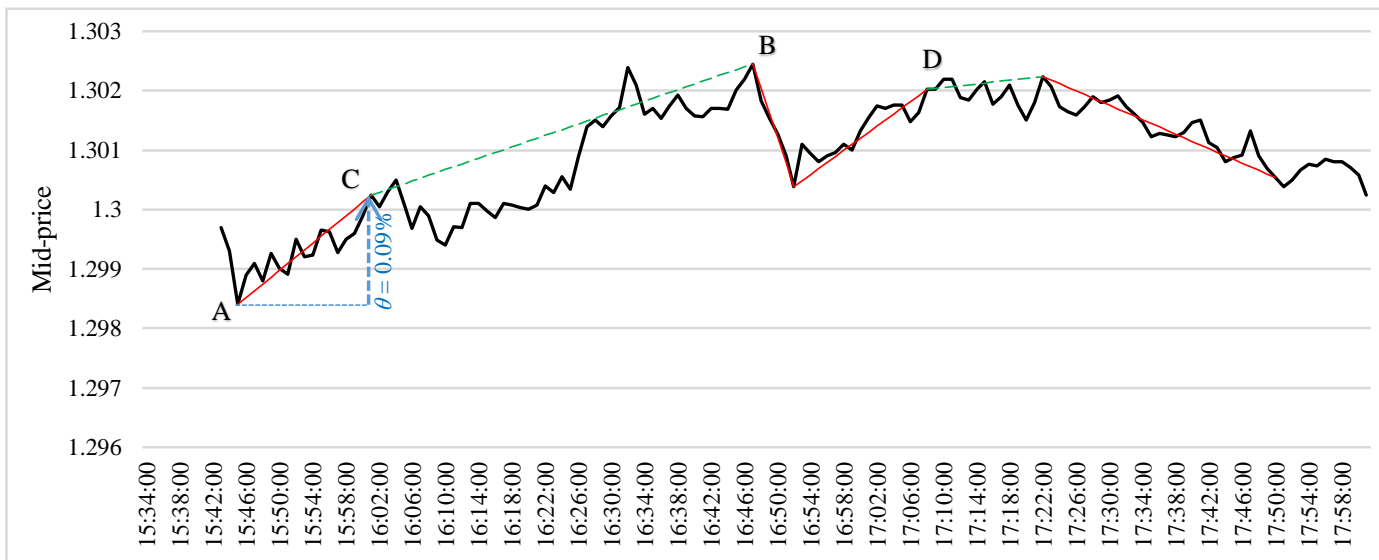

Fig. 1. An example of a DC-based summary. The black line indicates minute-by-minute EUR/USD mid-prices sampled from 15/12/2011 15:43:00 to 15/12/2011 18:01:00 (UK time). The red lines indicate Directional Change (DC) events and the green dashed lines indicate Overshoot $(\mathrm{OS})$ events. Here, $\theta=0.09 \%$.

\begin{tabular}{|c|c|}
\hline Name / Description & Notation \\
\hline Threshold & $\theta$ \\
\hline Current price & $\mathrm{P}_{\mathrm{c}}$ \\
\hline Price at extreme point: a price at which one trend ends and a new trend starts & $\mathrm{P}_{\mathrm{EXT}}$ \\
\hline $\begin{array}{l}\text { The highest price, during an uptrend's OS event, required to confirm that the market's } \\
\text { direction has changed to downtrend (i.e. to confirm a downtrend's DC event). }\end{array}$ & $\mathrm{P}_{\mathrm{DCC} \downarrow^{*}}=\mathrm{P}_{\mathrm{EXT}} \times(1-\theta)$ \\
\hline $\begin{array}{l}\text { The least price, during a downtrend's OS event, required to confirm that the market's } \\
\text { direction has changed to uptrend (i.e. to confirm an uptrend's DC event). }\end{array}$ & $\mathrm{P}_{\mathrm{DCC} \uparrow^{*}}=\mathrm{P}_{\mathrm{EXT}} \times(1+\theta)$ \\
\hline $\begin{array}{l}\text { Overshoot value (OSV) is defined at each price's observation during an OS event. Here, } \\
\mathrm{P}_{\mathrm{DCC} *}=\mathrm{P}_{\mathrm{DCC} \downarrow^{*}} \text { if the current trend is downtrend; otherwise } \mathrm{P}_{\mathrm{DCC} *}=\mathrm{P}_{\mathrm{DCC} \uparrow^{*}} \text {. }\end{array}$ & $\mathrm{OSV}=\left(\left(\mathrm{P}_{\mathrm{c}}-\mathrm{P}_{\mathrm{DCC}}\right) \div \mathrm{P}_{\mathrm{DCC}} *\right) \div \theta$ \\
\hline
\end{tabular}

uptrend, the $\mathrm{P}_{\text {EXT }}$ is the lowest price of the uptrend. In case of a downtrend, the $\mathrm{P}_{\mathrm{EXT}}$ is the highest price of the downtrend. The price movements between two consecutive $\mathrm{P}_{\mathrm{EXT}}$ form a trend. A trend comprises a directional change (DC) event and an overshoot (OS) event. Let $\mathrm{P}_{\mathrm{c}}$ be the current price of the market. A DC event is detected when we observe a price $P_{c}$ that satisfies inequality (1):

$$
\left|\left(\left(\mathrm{P}_{\mathrm{c}}-\mathrm{P}_{\mathrm{EXT}}\right) \div \mathrm{P}_{\text {EXT }}\right)\right| \geq \theta
$$

If inequality (1) holds, then the time at which the market traded at $\mathrm{P}_{\text {EXT }}$ is called an extreme point (e.g. points $\mathrm{A}$ and $\mathrm{B}$ in Fig.1), and the time at which the market trades at $P_{c}$ is called a DC confirmation point (e.g. points C and D in Fig.1). Each trend starts with an extreme point. A DC event starts with an extreme point and ends with a DC confirmation point. A DC event is recognized only in hindsight. It is recognized precisely at the DC confirmation point. In Fig. 1, point A represents the start of a DC event of an uptrend; and point B represents the start of a DC event of a downtrend. An OS event begins at a DC confirmation point (e.g. points $\mathrm{C}$ and $\mathrm{D}$ in Fig. 1) and ends at the succeeding extreme point [10]. The DC based summary of a given financial time series is the identification of the DC events and OS events, provided a threshold $\theta$. Fig. 1. shows an example of a DC based summary. Table 1 lists some essential notations used in this paper (adopted from [10]).

\section{BACKLASH ALgORITHM (BA): A DC-BASED TRADING STRATEGY}

In this section we introduce a new contrarian trading strategy named 'Backlash Algorithm', or BA for short. We describe two types of BA: BA-down and BA-up. For each of BA-down and BA-up, we provide two versions: static and dynamic. We provide the details of the static and dynamic versions of BA-down in Sections A. and B. respectively. BAup is pretty similar to BA-down. We will describe briefly the difference between BA-down and BA-up as we proceed in this section. In Section $\mathrm{C}$ we describe our money management approach.

\section{A. Static BA-down (SBA-down)}

We introduce a trading strategy named Static BA-down, or SBA-down for short. SBA-down is only applicable when the market is in downtrend (hence its name). SBA-down opens a long position when the value of OSV drops below a certain threshold, down_ind, during a downtrend's OS event. Note that the value of down_ind is the choice of the trader. SBA-down closes its position when the DC confirmation point of the next uptrend is confirmed. When SBA-down closes a position, it may generate profits or losses. SBA-down consists of two rules:

Rule 1: (open long position)

If (OSV $\leq$ down_ind) and (current trend is downtrend) and (current event is OS event) then generate buy signal 
Rule 2: (close position)

If ( $\left.\mathrm{Pc} \geq \mathrm{P}_{\mathrm{DCC}} \uparrow^{*}\right)$ and (current trend is uptrend) then generate sell signal

Similarly, we introduce the Static version of BA-up named SBA-up. SBA-up is the mirror of SBA-down. SBA-up opens a short position while the market is in uptrend and only if the value of OSV exceeds a certain threshold, named up_ind. SBAup generates buy signal when a new DC event of a downtrend is observed.

\section{B. Dynamic BA-down (DBA-down)}

When trading with SBA-down, we have no hint about how SBA-down will perform if we chose a value of down_ind arbitrarily. Suppose that SBA-down had performed well for a given value of down_ind during a trading period, named $x$, there is no guarantee that SBA-down will have same performance during another trading period, named $y$, using the same value of down_ind. These facts motivate the development of the dynamic version of SBA-down, namely DBA-down. DBAdown comprises two stages. In the first stage, DBA-down automatically determines the value of the parameter down_ind. For this purpose, DBA-down apply a procedure, named FIND_DOWN_IND, to a training dataset (i.e. training period) to determine the value of down_ind. In the second stage, DBAdown apply the same two rules of SBA-down to trade on an applied dataset (i.e. applied period) using the value of down_ind obtained using FIND_DOWN_IND.

The objective of the procedure FIND_DOWN_IND is to find a suitable value of the parameter down_ind for the applied period based on its performance during the training period. The procedure FIND_DOWN_IND returns one numerical variable, named best_down_ind. To determine best_down_ind, FIND_DOWN_IND apply SBA-down using 100 different values of down_ind (from - 0.01 to -1.00 , step size of - 0.01 ) to the training period. For each value of down_ind, we compute the profits obtained by applying SBA-down to the training period. Consequently, for a given training period we get 100 profits. best_down_ind is the value of down_ind under which SBA-down generates the highest profits during the training period. In the second stage of DBA-down, down_ind is assigned the value of best_down_ind. DBA-up is the dynamic version of SBA-up, as DBA-down to SBA-down. DBA-up has also two stages as in DBA-down. The first stage is to automatically compute the value of $u p \_i n d$ based using training period. The second stage consists of applying the same rules of SBA-up to the associated applied period.

\section{Money Management Approach}

In this section we describe our approach of money management. In this paper we apply the following approach to all versions of BA (i.e. SBA-down, SBA-up, DBA-down, and DBA-up). The entire amount of capital is used when SBAdown, or DBA-down, opens a long position. Similarly, SBAdown, or DBA-down, sells all available shares when closing a position. Likewise in case of SBA-up or DBA-up. When SBAup, or DBA-up, opens short position it sells all available shares. When it generate a buy signal, it uses the entire capital. Throughout this paper, when we apply any version of the
Backlash Algorithm, we make sure that no position is left open at the end of the trading period. In case we encounter an opened position at the end of the trading period, then we will not consider the last transaction when computing the results; we rollback the last transaction instead. In other words, we do not count this last trade when measuring any of the evaluation metrics (to be introduced later in Section IV).

To summarize, in Section III we introduce a new contrarian trading strategy named Backlash Algorithm, or BA for short. BA has two types: BA-down and BA-up. Each type has two versions: static and dynamic. We also introduce the money management approach adopted in our experiments throughout this paper.

\section{EXPERIMENTS}

We test our proposed trading strategy in the foreign exchange market. We use a rolling windows approach to evaluate the performance of BA. This section is organized as follow: In Section A, we describe how we compose a set of rolling windows using EUR/CHF mid-prices series. In Section $\mathrm{B}$, we list the evaluation metrics used to assess the performance of the proposed strategy. The objective of Section $\mathrm{C}$ is to evaluate the performance of the static versions SBA-down and SBA-up. The objective of Section $D$ is to evaluate the performance of the dynamic versions DBA-down and DBA-up. In Section $\mathrm{E}$ we test whether the profits generated by our strategy can be affected by the value of theta $(\theta)$. The objective of Section $\mathrm{F}$ is to test the profitability of our trading strategy works in another asset. For this purpose, we apply BA to EUR/USD exchange rates.

\section{A. Preparing the Datasets and the Rolling Windows}

In this paper we use a rolling window approach to test the profitability of our proposed trading strategies. In this section we describe how to prepare these rolling windows using the initial dataset. Our initial dataset is composed of 31 months of minute-by-minute EUR/CHF mid-prices sampled from 1/1/2013 00:01:00 to 31/07/2015 23:59:00 (UK time).

\section{1) Producing DC Analysis for the Datasets}

We apply the Directional change (DC) analysis to the initial dataset of EUR/CHF mid-prices. Given a threshold $\theta$, the DC analysis encompasses the identification of all DC events and OS events in the initial dataset and the computation of the variables OSV and $\mathrm{P}_{\mathrm{DCC}} *$ (Table 1). We set, arbitrarily, $\theta=0.1 \%$ and we apply the DC analysis to the initial dataset of EUR/CHF. Let DCO.1 be the dataset exemplified in Table 2. DC0.1 comprises the date, time and the price of each observation of the initial dataset. In Table 2, the column 'Event Type' marks the occurrence of any DC or OS event that starts at the corresponding observation. The columns 'OSV' and ' $\mathrm{P}_{\mathrm{DCC}}$ ' refer to the variables already defined in Table 1.

\section{2) Composing Rolling Windows Based on the Dataset} DCO.1

We use a rolling window approach (Fig. 2) to evaluate the performance of our proposed trading strategies. As the dataset DC0.1 cover 31 months, we compose seven rolling windows; 
Table 2: An example of a DC analysis using EUR/CHF mid-prices sampled from 31/7/2015 11:20:00 to 31/7/2015 11:31:00 (UK time) ( $\theta=0.1 \%$ ). The values of $\mathrm{P}_{\mathrm{DCC} *}$ and OSV are rounded to 5 decimal places.

\begin{tabular}{cccccc} 
Date & Time & Mid-price & Event type & PoCC* & OSV \\
\hline $31 / 7 / 2015$ & $11: 20: 00$ & 1.06336 & (start DC DOWNTREND ) & 0 & 0 \\
\hline $31 / 7 / 2015$ & $11: 21: 00$ & 1.06290 & & 0 & 0 \\
\hline $31 / 7 / 2015$ & $11: 22: 00$ & 1.06333 & & 0 & 0 \\
\hline $31 / 7 / 2015$ & $11: 23: 00$ & 1.06320 & & 0 & 0 \\
\hline $31 / 7 / 2015$ & $11: 24: 00$ & 1.06258 & & 0 & 0 \\
\hline $31 / 7 / 2015$ & $11: 25: 00$ & 1.06230 & (start OS DOWNTREND) & 0 & 0 \\
\hline $31 / 7 / 2015$ & $11: 26: 00$ & 1.06241 & & 0 & 0 \\
\hline $31 / 7 / 2015$ & $11: 27: 00$ & 1.06242 & & 1.06299 & -0.70285 \\
\hline $31 / 7 / 2015$ & $11: 28: 00$ & 1.06155 & 0 & -0.74992 \\
\hline $31 / 7 / 2015$ & $11: 29: 00$ & 1.06150 & & 0 & -0.37338
\end{tabular}

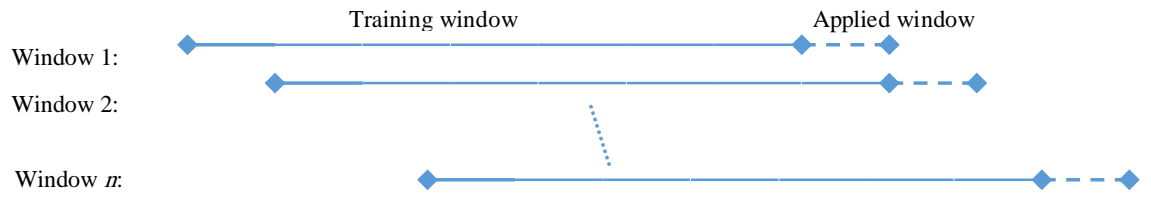

Fig. 2. Illustration of $n$ rolling windows. The dashed lines represent the applied windows.

each of which comprises a training window (length of 24 months) and an applied window (length of 1 month). The length of the training and applied windows are set arbitrarily. Let $R W D C 0.1$ represents the set of these seven rolling windows. Note that we measure the length of training and applied windows as function of months; not as fixed number of days. For example the training period of the second rolling window lasts from $1 / 2 / 2013$ to $31 / 1 / 2015$ (i.e. 24 months). The associated applied window last from 1/2/2015 00:01:00 to 28/2/2015 23:59:00 (i.e. the month of February 2015). Note that although our initial dataset, of EUR/CHF, was sampled as time series (with interval of one minute in our case), but the BA's trading rules (presented in Section III) are based on variables (e.g. OSV and $\mathrm{P}_{\mathrm{DCC}}$ ) those originate from the $\mathrm{DC}$ analysis.

\section{B. Evaluation Metrics}

We chose the following metrics to measure the performance of our proposed trading strategy. These metrics have been reported as necessary to assess a given trading strategy [16].

- Total profit: The total profit symbolizes the bottom line for a trading system over a definite period of time. The total profit is computed by removing the gross loss of all losing trades from the gross profit of all winning trades.

- Profit factor: The profit factor is defined as the gross profit divided by the gross loss for the entire trading period. This metric measure the amount of profit per unit of risk, with values greater than one signifying a profitable system.

- Max drawdown (\%): The drawdown is define as the difference, in percentage, between the highest profit, previous to the current time point, and the current profit value. The Maximum Drawdown (MDD) is the largest drawdown observed during a specific trading period.
- Profitability percentage: This metric is calculated by dividing the number of winning trades by the total number of trades for a specified trading period.

- Sortino ratio [17]: The downside risk is defined as the standard deviation of negative asset return, called downside deviation. The Sortino ratio, (2), uses the downside risk to measure the risk associated to a given investment. In (2), the 'return' represents the profits generated by a given trading strategy and the 'target return' is the minimum acceptable return (MAR).

Sortino ratio $=($ return - target return $) \div$ downside risk

\section{Experiment 1: Evaluation of the Static Versions of BA}

The objective of this section is two-fold. Firstly, we want to evaluate the performance of the static versions of BA (i.e. SBAup and SBA-down). Secondly, we want to examine whether there exists a particular value of the parameters, down_ind and up_ind, for which SBA-down and SBA-up will have the best performance consistently (i.e. for each rolling window of $R W D C 0.1$ in our case). In all of the following experiments, we apply the money management approach described in Section III.

3) Experiment 1.1: Measuring the Performance of SBAdown

The objective of this experiment is to evaluate the performance of SBA-down. For this purpose we apply SBAdown to each applied window in RWDC0.1 using 100 different values of down_ind (from -0.01 to -1.00 , step size of -0.01 ). Consequently, for each applied window we will have 100 profits (each profits correspond to one distinct value of down_ind). For simplicity, we consider the profits as indicator of the performance of SBA-down. Therefore, for each applied window we report the highest and the lowest generated profits together with the other defined evaluation metrics in Section IV. 


\section{4) Experiment 1.2: Measuring the Performance of SBA-} up

The objective of this experiment is to evaluate the performance of SBA-up. Here, we apply the same approach as in Experiment 1.1. We apply SBA-up to each applied window in $R W D C 0.1$ using 100 different values of $u p \_$ind (from 0.01 to 1.00 , with step size of 0.01 ). For each applied window, we compute the generated 100 profits. Then, we report the highest and the lowest profits in addition to the introduced evaluation metrics for each applied window.

5) Is There One Optimal Value for the Parameters down_ind and up_ind?

The objective of this section is to investigate whether there is a specific value of the parameters, down_ind, and up_ind, under which SBA-down, and SBA-up, will produce the best performance for all applied periods. This can be done by observing and analyzing the values of parameters down_ind and up_ind corresponding to the highest profits generated by SBAdown and SBA-up in Experiments 1.1. and 1.2. $B A$

D. Experiment 2: Evaluation of the Dynamic Versions of

The objective of this experiment is to evaluate the performance of DBA-down and DBA-up. Therefore we apply each of them to the seven rolling windows of $R W D C 0.1$. For each of DBA-down and DBA-up, we measure the metrics reported in Section IV.

Furthermore, as part of the evaluation of dynamic BA, we compare the performance of the static versions to the performance of the dynamic versions of BA. Bear in mind that when trading with the static versions the trader must chose the values of the parameters down_ind and up_ind. Consider that a trader assigns a random value to the parameter down_ind, or up_ind, when trading with the static version SBA-down, or SBA-up. The question is: What is the probability that the dynamic version DBA-down, or DBA-up, will produce higher profits than the static version SBA-down, or SBA-up? Let $\alpha$ denote this probability. As there is no evidence that the performance of BA-down and BA-up are equals, we estimate $\alpha$ for each of BA-down and BA-up.

To compute $\alpha$, we estimate the performance of the static versions using a set of random chosen values for input parameters down_ind and up_ind. For this purpose, we trade with SBA-down on RWDC0.1. 10,000 times. Each time, we trade with SBA-down on each applied windows in RWDC0.1. Each time, and for each applied window, we assign a new random value to the parameter down_ind. In other words, each time we trade with SBA-down using 7 random values of down_ind; each random value is used for one applied window. Each time, we measure the profits generated by SBA-down. Hence, we obtain 10,000 profits. Each profits corresponds to one trade with SBA-down on the seven rolling windows of $R W D C 0.1 . \alpha$ can be calculated as the fraction of how many of these 10,000 profits are less than the profits generated by the dynamic version, DBA-down. Similarly, we apply SBA-up to the applied windows of $R W D C 0.110,000$ times with randomly picked values for parameter up_ind. Each time and for each applied window, we assign a new random value to the parameter $u p \_i n d$. We obtain another 10,000 profits. Again, $\alpha$ is computed as the fraction of how many of these 10,000 profits are less than the profits generated by DBA-up.

\section{$B A$}

E. Experiment 3: The Impact of $\theta$ on the Performance of

The objective of this experiment is to test whether the generated profits by DBA-down and DBA-up may vary if we chose another value of $\theta$. For this purpose we run a new DC analysis with $\theta=0.07 \%$, chosen arbitrarily, to the same initial dataset of EUR/CHF. Based on this DC analysis, we compose a new set of seven rolling windows. Let $R W D C 0.07$ represents a new set comprises these seven rolling windows. Each rolling window comprises a training window of 24 months and an applied window of 1 month. We apply DBA-down and DBAup to each rolling window in RWDC0.07. Note that the static versions will not be applied in this experiment. This is because a trader, eventually, will not trade with the static versions unless he/she has evidence about which value should be assigned to the parameters down_ind or up_ind.

\section{F. Experiment 4: Applying DBA-up and DBA-down to} Other Asset

The objective of this experiment is to evaluate the profitability of our strategy in other market. To this end, we apply DBA-down and DBA-up to EUR/USD mid-prices series. The EUR/USD is sampled minute-by-minute for 31 months from 1/1/2013 00:01:00 to 31/7/2015 23:59:00. We run a new DC analysis, with $\theta=0.1 \%$, to EUR/USD. Using this DC analysis, we compose seven rolling windows. Each rolling window comprises a training window of 24 months and an applied window of 1 month. We apply DBA-down and DBAup to each window in this set. As in Experiment 3, the static versions will not be applied in this experiment.

\section{RESULTS AND ANALYSIS}

\section{A. Experiment 1: Evaluation of the Static Versions of $B A$}

Experiment 1 has two objectives. The first objective is to evaluate SBA-down and SBA-up. The second objective is to check if it is possible to have one single value of down_ind and $u p \_i n d$ for which SBA-down and SBA-up will generate the best possible profits regularly.

\section{1) Experiments 1.1 and 1.2.: Measuring the}

\section{Performance of SBA-down and SBA-up}

In this section we evaluate the performance of SBA-down and SBA-up. We apply both SBA-down and SBA-up to the seven applied windows of RWDC0.1. Tables 3 and 4 show respectively the best and the worst performance of SBA-down when applied to the applied window of $R W D C 0.1$ (see Section IV). These tables includes the following metrics: Profits, profit factor, maximum drawdown, and profitability percentage. Tables 3 and 4 have, almost, the same interpretation. The first column defines the applied windows (i.e. the trading period). The column 'Total number of trades' is the number of trades executed by SBA-down during the specified applied window. In Table 3 the column 'down ind' designs the value of down_ind which correspond to the highest profits that could be 
generated by SBA-down during the specified applied window. In Table 4, however, the column 'down ind' designs the value of down_ind which correspond to the lowest profits that could be generated by SBA-down during the specified applied window.

At the beginning of the first applied window, i.e. January 2015, SBA-down starts with capital $=1,000,000$; which represents the initial, hypothetically, invested amount of money. Here, the total profits ${ }^{1}$ is obtained by summing up the profits of the seven applied periods in both Tables 3 and 4. By doing so, we get $80.42 \%$ and $37.30 \%$; which represents respectively the best and the worst total profits possibly obtained by applying SBA-down to the applied windows of RWDC0.1.

Likewise, Tables 5 and 6 report, respectively, the best and the worst performance of SBA-up when it's applied to the seven applied windows of RWDC0.1. The value in the column ' $u p \_$ind' in Tables 5 and 6 represent the value of $u p \_i n d$ which correspond, respectively, to the highest and the lowest profits that could be generated by SBA-up during the specified applied window. In case of SBA-up, we assume that we start trading with an amount of shares of market value equal to $1,000,000$. Again, we sum up the profits in Tables 5 and 6 . In the best case, SBA-up generates a total profits of $64.78 \%$. In the worst case, SBA-up generates a total profits of $17.77 \%$.

Table 3: The best possible performance of applying SBA-down to RWDC0.1. The results reported here correspond to the applied windows only.

\begin{tabular}{ccccccc}
$\begin{array}{c}\text { Applied } \\
\text { window }\end{array}$ & down_ind & $\begin{array}{c}\text { Profits } \\
(\boldsymbol{\%})\end{array}$ & $\begin{array}{c}\text { Profit factor } \\
\text { (profit } \div \text { loss) }\end{array}$ & $\begin{array}{c}\text { Total number } \\
\text { of trades }\end{array}$ & $\begin{array}{c}\text { Max } \\
\text { drawdown (\%) }\end{array}$ & $\begin{array}{c}\text { Profitability } \\
\text { percentage }(\%)\end{array}$ \\
\hline Jan 2015 & -0.84 & 10.72 & 1.32 & 382 & -10.85 & 69.4 \\
\hline Feb 2015 & -0.43 & 15.63 & 3.28 & 284 & -0.76 & 76.4 \\
\hline Mar 2015 & -0.01 & 12.65 & 2.11 & 328 & -0.67 & 70.1 \\
\hline Apr 2015 & -0.04 & 7.80 & 1.99 & 198 & -0.47 & 71.7 \\
\hline May 2015 & -0.07 & 8.12 & 2.04 & 192 & -0.68 & 72.9 \\
\hline Jun 2015 & -0.14 & 10.38 & 1.91 & 234 & -1.31 & 76.1 \\
\hline Jul 2015 & -0.39 & 15.11 & 3.46 & 180 & -0.59 & 84.4
\end{tabular}

Table 4: The worst possible performance of applying SBA-down to RWDC0.1. The results reported here correspond to the applied windows only.

\begin{tabular}{ccccccc}
$\begin{array}{c}\text { Applied } \\
\text { window }\end{array}$ & down_ind & $\begin{array}{c}\text { Profits } \\
(\boldsymbol{\%})\end{array}$ & $\begin{array}{c}\text { Profit factor } \\
\text { (profit } \div \text { loss) }\end{array}$ & $\begin{array}{c}\text { Total number } \\
\text { of trades }\end{array}$ & $\begin{array}{c}\text { Max } \\
\text { drawdown }(\boldsymbol{\%})\end{array}$ & $\begin{array}{c}\text { Profitability } \\
\text { percentage }(\%)\end{array}$ \\
\hline Jan 2015 & -0.11 & 3.77 & 1.09 & 513 & -11.75 & 67.4 \\
\hline Feb 2015 & -1.00 & 9.80 & 3.81 & 164 & -0.70 & 82.9 \\
\hline Mar 2015 & -1.00 & 8.19 & 3.57 & 142 & -0.37 & 76.8 \\
\hline Apr 2015 & -0.83 & 3.86 & 2.24 & 96 & -0.45 & 69.8 \\
\hline May 2015 & -1.00 & 2.87 & 2.08 & 73 & -0.49 & 72.6 \\
\hline Jun 2015 & -1.00 & 3.20 & 1.69 & 97 & -0.96 & 75.3 \\
\hline Jul 2015 & -1.00 & 5.61 & 3.46 & 82 & -0.40 & 80.5
\end{tabular}

Table 5: The best possible performance of applying SBA-up to $R W D C 0.1$. The results reported here correspond to the applied windows only.

\begin{tabular}{ccccccc}
$\begin{array}{c}\text { Applied } \\
\text { window }\end{array}$ & up_ind & $\begin{array}{c}\text { Profits } \\
(\boldsymbol{\%})\end{array}$ & $\begin{array}{c}\text { Profit factor } \\
\text { (profit } \div \text { loss) }\end{array}$ & $\begin{array}{c}\text { Total number } \\
\text { of trades }\end{array}$ & $\begin{array}{c}\text { Max } \\
\text { drawdown }(\boldsymbol{\%})\end{array}$ & $\begin{array}{c}\text { Profitability } \\
\text { percentage }(\boldsymbol{\%})\end{array}$ \\
\hline Jan 2015 & 0.73 & -4.08 & 0.88 & 389 & -14.04 & 65.6 \\
\hline Feb 2015 & 0.04 & 10.60 & 1.98 & 371 & -0.76 & 67.7 \\
\hline Mar 2015 & 0.09 & 15.13 & 3.40 & 316 & -0.40 & 75.3 \\
\hline Apr 2015 & 0.11 & 7.80 & 1.99 & 200 & -1.93 & 76.0 \\
\hline May 2015 & 0.04 & 11.65 & 3.17 & 200 & -0.49 & 78.5 \\
\hline Jun 2015 & 0.01 & 11.00 & 1.92 & 268 & -0.95 & 72.8 \\
\hline Jul 2015 & 0.15 & 12.68 & 2.43 & 219 & -0.77 & 76.7
\end{tabular}

Table 6: The worst possible performance of applying SBA-up to $R W D C 0.1$. The results reported here correspond to the applied windows only.

\begin{tabular}{ccccccc}
$\begin{array}{c}\text { Applied } \\
\text { window }\end{array}$ & up_ind & $\begin{array}{c}\text { Profits } \\
(\boldsymbol{\%})\end{array}$ & $\begin{array}{c}\text { Profit factor } \\
\text { (profit } \div \text { loss) }\end{array}$ & $\begin{array}{c}\text { Total number } \\
\text { of trades }\end{array}$ & $\begin{array}{c}\text { Max } \\
\text { drawdown (\%) }\end{array}$ & $\begin{array}{c}\text { Profitability } \\
\text { percentage }(\boldsymbol{\%})\end{array}$ \\
\hline Jan 2015 & 0.89 & -6.38 & 0.80 & 344 & -14.05 & 63.1 \\
\hline Feb 2015 & 0.90 & 6.56 & 2.46 & 190 & -0.59 & 72.6 \\
\hline Mar 2015 & 1.00 & 6.73 & 4.13 & 133 & -0.29 & 82.0 \\
\hline Apr 2015 & 0.98 & 1.68 & 1.50 & 78 & -1.34 & 71.8 \\
\hline May 2015 & 1.00 & 3.69 & 2.95 & 75 & -0.58 & 76.0 \\
\hline Jun 2015 & 0.93 & 2.83 & 1.64 & 113 & -0.85 & 70.8 \\
\hline Jul 2015 & 0.98 & 2.66 & 1.90 & 85 & -0.67 & 76.5
\end{tabular}

Table 7: The downside risk and Sortino ratio of the different versions of BA.

\begin{tabular}{cccccccc} 
& $\begin{array}{c}\text { SBA-down } \\
\text { (worst case) }\end{array}$ & $\begin{array}{c}\text { SBA-down } \\
\text { (best case) }\end{array}$ & DBA-down & $\begin{array}{c}\text { SBA-up } \\
\text { (worst case) }\end{array}$ & $\begin{array}{c}\text { SBA-up } \\
\text { (best case) }\end{array}$ & DBA-up \\
\hline Downside risk & 0 & 0 & 0 & 2.4429 & 1.5736 & 1.8722 \\
\hline Sortino ratio & NA & NA & NA & 7.0272 & 40.7897 & 31.5266
\end{tabular}

${ }^{1}$ The profits reported in this paper do not include the transaction costs. 
The downside risk and Sortino ratio of the different versions of BA are shown in Table 7. The minimum acceptable return (MAR) is set to $1 \%$ per annum. In addition, we apply the buy and hold approach to the same seven applied windows of $R W D C 0.1$. We buy on 1/1/2015 00:01:00 with price of 1.20279; then we sell on 7/31/2015 23:59:00 with price of 1.06120. The profits generated by the buy-and-hold would be: $100 \times(1.20279$ $-1.06120) / 1.20279=-14.16 \%$.

Based on the results reported in Tables 3 and 4, the highest and lowest profits that could be generated by SBA-down are $80.41 \%$ and $37.31 \%$ respectively. Whereas, the highest and lowest profits that could be generated by SBA-up are $64.78 \%$ and $17.77 \%$ respectively. As can be noted in Table 7 , the downside risk of SBA-down is null but the downside risk of SBA-up is not. These observations suggest that SBA-up and SBA-down may have different performance during same trading period.

To summarize, the results of Experiment 1 suggest that SBA-down and SBA-up are able to generate a total profit of up to $80 \%$ and $64 \%$, respectively, within the specified seven months; whereas the buy-and-hold approach generates profits of $-14 \%$ during the same trading period. This highlights the importance of both versions of SBA.

2) Is there One Optimal Value for the Parameters down_ind and up_ind?

The objective of this section is to investigate whether there exists a specific value of the parameters down_ind, and up_ind, for which SBA-down, and SBA-up, will generate the highest profits consistently. Based on the results of Experiments 1.1 and 1.2., we highlight the following observations:

1. Concerning SBA-down: Based on Tables 3 and 4, we conclude that determining the value of down_ind is very critical. Because for a small value of down_ind SBA-down may yield the best profits (as in March 2015 , for down_ind $=-0.01$ ) or it may yield the worst profits (as in January 2015, for down_ind $=-0.11$ ). Similarly, for a large value of down_ind SBA-down may yield the best profits (as in January 2015, for up_ind $=0.84$ ) or it may yield the worst profits (as in February 2015, for $u p \_i n d=1.00$ ).
2. Concerning SBA-up: In Tables 5, we note that SBAup may generates the best profits using large value of up_ind (as in January 2015, for up_ind $=0.73$ ) or using small value of up_ind (as in June 2015, for up_ind = $0.01)$.

Observations 1 and 2 suggest that the optimal performance of SBA-down and SBA-up can be achieved sometimes using small values of down_ind and $u p \_i n d$; and using large value of down_ind and up_ind on other times. Thus, there is no specific value for these parameters for which SBA-down and SBA-up will have the best performance consistently. $B A$

B. Experiment 2: Evaluation of the Dynamic Versions of

The objective of these experiments is to evaluate the performance of DBA-down and DBA-up. We apply each of DBA-down and DBA-up to each of the seven rolling windows of $R W D C 0.1$. For each of DBA-down and DBA-up, we start with 1,000,000 as initial invested capital. Tables 8 and 9 report, respectively, the evaluation of the performance of DBA-down and DBA-up. Tables 8 and 9 have same interpretation as Tables 4 and 5 respectively. The sum of the profits generated by DBAdown and DBA-up, in Tables 8 and 9, are $63.61 \%$ and $59.60 \%$ respectively. The downside risk and Sortino ratio of DBAdown and DBA-up are reported in Table 7. In this experiment, DBA-down generates 2008 trades; of which 1445 are winning trades (profit factor is 2.57). Whereas, DBA-up generates 2104 trades; of which 1486 are winning trades (profit factor is 2.40).

As a second approach to evaluate DBA-down and DBA-up we compare them to SBA-down and SBA-up with randomly picked parameters. We apply each of SBA-down and SBA-up 10,000 times to the applied windows of $R W D C 0.1$ using randomly picked value of parameters down_ind and up_ind. We define $\alpha$ as the fraction of how many of these 10,000 profits are less than the profits obtained by DBA-down and DBA-up (reported in Tables 8 and 9). In case of SBA-down we have $\alpha=$ $88 \%$ (i.e. the probability that DBA-down outperforms SBAdown with randomly picked parameter is $88 \%$ ). In case of SBAup, the probability that DBA-up outperforms SBA-up with randomly picked parameter is $\alpha=97 \%$.

\begin{tabular}{|c|c|c|c|c|c|c|}
\hline $\begin{array}{l}\text { Applied } \\
\text { window }\end{array}$ & down_ind & $\begin{array}{c}\text { Profits } \\
(\%)\end{array}$ & $\begin{array}{l}\text { Profit factor } \\
\text { (profit } \div \text { loss) }\end{array}$ & $\begin{array}{l}\text { Total number } \\
\text { of trades }\end{array}$ & $\begin{array}{c}\text { Max } \\
\text { drawdown }(\%)\end{array}$ & $\begin{array}{c}\text { Profitability } \\
\text { percentage }(\%)\end{array}$ \\
\hline Jan 2015 & -0.11 & 3.77 & 1.09 & 513 & -11.75 & 67.4 \\
\hline Feb 2015 & -0.10 & 13.56 & 2.45 & 345 & -0.90 & 73.9 \\
\hline Mar 2015 & -0.10 & 11.01 & 2.12 & 307 & -0.81 & 68.7 \\
\hline Apr 2015 & -0.09 & 5.99 & 1.86 & 186 & -0.47 & 71.0 \\
\hline May 2015 & -0.09 & 7.36 & 2.03 & 190 & -0.68 & 72.6 \\
\hline Jun 2015 & -0.09 & 8.94 & 1.83 & 237 & -1.39 & 75.5 \\
\hline Jul 2015 & -0.09 & 12.98 & 2.78 & 218 & -0.64 & 78.4 \\
\hline
\end{tabular}

Table 9. Results of applying DBA-up to RWDC0.1. The results reported here correspond to the applied windows only.

\begin{tabular}{ccccccc}
$\begin{array}{c}\text { Applied } \\
\text { window }\end{array}$ & up_ind & $\begin{array}{c}\text { Profits } \\
(\boldsymbol{\%})\end{array}$ & $\begin{array}{c}\text { Profit factor } \\
\text { (profit } \div \text { loss) }\end{array}$ & $\begin{array}{c}\text { Total number } \\
\text { of trades }\end{array}$ & $\begin{array}{c}\text { Max } \\
\text { drawdown (\%) }\end{array}$ & $\begin{array}{c}\text { Profitability } \\
\text { percentage }(\%)\end{array}$ \\
\hline Jan 2015 & 0.17 & -4.87 & 0.88 & 504 & -14.74 & 63.1 \\
\hline Feb 2015 & 0.03 & 10.32 & 1.95 & 372 & -8.68 & 67.7 \\
\hline Mar 2015 & 0.03 & 14.71 & 3.21 & 324 & -0.44 & 74.4 \\
\hline Apr 2015 & 0.03 & 7.37 & 1.91 & 209 & -1.71 & 75.6 \\
\hline May 2015 & 0.03 & 11.42 & 3.14 & 201 & -0.37 & 78.1 \\
\hline Jun 2015 & 0.03 & 10.21 & 1.87 & 265 & -0.94 & 72.5 \\
\hline Jul 2015 & 0.03 & 10.44 & 2.08 & 229 & -0.81 & 73.4 \\
\hline
\end{tabular}


To summarize, in Experiment 1 we reported that the weakness of the SBA is that it is hard for a trader to guess the best value of the parameters down_ind and up_ind. In Experiment 2, we show that the dynamic versions can outperform the static versions in more than $88 \%$ of the cases (provided we assign random values to these parameters when trading with the static versions). The advantage of DBA is that it computes automatically the value of these parameters.

\section{Experiment 3: Impact of Threshold $\theta$ on the} Performance of DBA-down and DBA-up

The objective of this experiment is to test whether the performances of DBA-down and DBA-up can be affected by the value of $\theta$. In Experiment 2, we used $\theta=0.1 \%$. In Experiment 3, we run DC analysis to the initial dataset of EUR/CHF with $\theta=0.07 \%$. The results of applying DBA-down and DBA-up to each rolling windows are shown in Tables 10 and 11 respectively. Again, we start with 1,000,000 as initial invested capital. We compare these results with the results obtained by DBA-up and DBA-down in Experiment 2.

In this experiment, the sum of all profits generated by DBAdown, reported in column 3 of Table 10, is $101.12 \%$. This is about $59 \%$ larger than the total profits generated by DBA-down in Experiment 2. which is $63.61 \%$ (the sum of profits in column 3 in Table 8). On the other hand, in this experiment the total profits generated by DBA-up, in Table 11, is $145.00 \%$. This is about $246 \%$ larger than the total profits generated by DBA-up in Experiment 2. which is $59.60 \%$ (see the sum of profits in column 3 in Table 9). These notes indicate that the value of $\theta$ can affect the performance of BA. In this experiment, DBAdown generates 3320 trades; of which 2343 are winning trades (profit factor is 2.40). Whereas, DBA-up generates 3534 trades; of which 2588 are winning trades (profit factor is 2.74).

\section{Experiment 4: Applying DBA-up and DBA-down to} Other Asset

The objective of this section is to investigate the profitability of BA in another market. Therefore, we apply DBA-up and DBA-down to another currency pairs: EUR/USD. We apply the same approach, as in Section IV, to compose a new set of rolling windows based on DC analysis of EUR/USD. We start with 1,000,000 as initial invested capital.

Table 10. Results of applying DBA-down to the 7 rolling windows of $R$ WDC0.07. The results reported here correspond to the applied windows only.

\begin{tabular}{ccccccc}
$\begin{array}{c}\text { Applied } \\
\text { window }\end{array}$ & down_ind & $\begin{array}{c}\text { Profits } \\
(\boldsymbol{\%})\end{array}$ & $\begin{array}{c}\text { Profit factor } \\
\text { (profit } \div \text { loss) }\end{array}$ & $\begin{array}{c}\text { Total number } \\
\text { of trades }\end{array}$ & $\begin{array}{c}\text { Max } \\
\text { drawdown (\%) }\end{array}$ & $\begin{array}{c}\text { Profitability } \\
\text { percentage (\%) }\end{array}$ \\
\hline Jan 2015 & -0.03 & 0.44 & 1.01 & 713 & -12.27 & 66.2 \\
\hline Feb 2015 & -0.15 & 10.26 & 1.69 & 525 & -0.97 & 70.5 \\
\hline Mar 2015 & -0.02 & 12.46 & 1.86 & 541 & -0.88 & 70.8 \\
\hline Apr 2015 & -0.03 & 40.65 & 5.10 & 375 & -0.63 & 71.2 \\
\hline May 2015 & -0.03 & 9.16 & 1.93 & 339 & -0.63 & 70.8 \\
\hline Jun 2015 & -0.03 & 12.47 & 1.84 & 429 & -1.48 & 73.7 \\
\hline Jul 2015 & -0.04 & 15.68 & 2.58 & 398 & -0.66 & 74.1
\end{tabular}

Table 11. Results of applying DBA-up to the 7 rolling windows of $R W D C 0.07$. The results reported here correspond to the applied windows only.

\begin{tabular}{ccccccc}
$\begin{array}{c}\text { Applied } \\
\text { window }\end{array}$ & up_ind & $\begin{array}{c}\text { Profits } \\
(\%)\end{array}$ & $\begin{array}{c}\text { Profit factor } \\
\text { (profit } \div \text { loss) }\end{array}$ & $\begin{array}{c}\text { Total number } \\
\text { of trades }\end{array}$ & $\begin{array}{c}\text { Max } \\
\text { drawdown (\%) }\end{array}$ & $\begin{array}{c}\text { Profitability } \\
\text { percentage (\%) }\end{array}$ \\
\hline Jan 2015 & 0.06 & 9.74 & 1.19 & 790 & -10.83 & 68.0 \\
\hline Feb 2015 & 0.08 & 43.62 & 3.75 & 567 & -0.58 & 72.5 \\
\hline Mar 2015 & 0.08 & 22.78 & 2.80 & 559 & -0.49 & 75.1 \\
\hline Apr 2015 & 0.07 & 17.11 & 2.46 & 399 & -1.13 & 78.7 \\
\hline May 2015 & 0.07 & 15.88 & 2.55 & 348 & -0.53 & 76.4 \\
\hline Jun 2015 & 0.02 & 16.59 & 1.80 & 447 & -1.11 & 73.6 \\
\hline Jul 2015 & 0.01 & 19.28 & 2.06 & 424 & -1.08 & 73.3 \\
\hline
\end{tabular}

Table 12. Results of applying DBA-down to the 7 rolling windows of EUR/USD. The results reported here correspond to the applied windows only.

\begin{tabular}{ccccccc}
$\begin{array}{c}\text { Applied } \\
\text { window }\end{array}$ & down_ind & $\begin{array}{c}\text { Profits } \\
(\boldsymbol{\%})\end{array}$ & $\begin{array}{c}\text { Profit factor } \\
\text { (profit } \div \text { loss) }\end{array}$ & $\begin{array}{c}\text { Total number } \\
\text { of trades }\end{array}$ & $\begin{array}{c}\text { Max } \\
\text { drawdown (\%) }\end{array}$ & $\begin{array}{c}\text { Profitability } \\
\text { percentage (\%) }\end{array}$ \\
\hline Jan 2015 & -0.01 & -2.74 & 0.83 & 301 & -3.62 & 61.8 \\
\hline Feb 2015 & -0.08 & 2.53 & 1.30 & 221 & -1.03 & 63.8 \\
\hline Mar 2015 & -0.30 & -2.69 & 0.84 & 313 & -3.89 & 62.9 \\
\hline Apr 2015 & -0.31 & 3.78 & 1.31 & 295 & -2.21 & 65.1 \\
\hline May 2015 & -0.35 & 5.50 & 1.55 & 283 & -1.04 & 67.5 \\
\hline Jun 2015 & -0.41 & 5.50 & 1.43 & 284 & -1.43 & 68.3 \\
\hline Jul 2015 & -0.35 & 0.78 & 1.07 & 222 & -1.26 & 59.5 \\
\hline
\end{tabular}

Table 13. Results of applying DBA-up to the 7 rolling windows of EUR/USD. The results reported here correspond to the applied windows only.

\begin{tabular}{ccccccc}
$\begin{array}{c}\text { Applied } \\
\text { window }\end{array}$ & up_ind & $\begin{array}{c}\text { Profits } \\
(\%)\end{array}$ & $\begin{array}{c}\text { Profit factor } \\
\text { (profit } \div \text { loss) }\end{array}$ & $\begin{array}{c}\text { Total number } \\
\text { of trades }\end{array}$ & $\begin{array}{c}\text { Max } \\
\text { drawdown (\%) }\end{array}$ & $\begin{array}{c}\text { Profitability } \\
\text { percentage (\%) }\end{array}$ \\
\hline Jan 2015 & 0.01 & 5.64 & 1.46 & 303 & -1.37 & 65.7 \\
\hline Feb 2015 & 0.09 & 4.15 & 1.49 & 216 & -0.92 & 67.1 \\
\hline Mar 2015 & 0.10 & 1.67 & 1.09 & 355 & -4.68 & 65.9 \\
\hline Apr 2015 & 0.10 & 0.20 & 1.01 & 337 & -2.87 & 62.6 \\
\hline May 2015 & 0.11 & 7.24 & 1.53 & 319 & -1.20 & 68.7 \\
\hline Jun 2015 & 0.10 & 4.77 & 1.26 & 360 & -1.01 & 65.8 \\
\hline Jul 2015 & 0.11 & 1.95 & 1.13 & 252 & -2.39 & 65.5 \\
\hline
\end{tabular}


The results of applying DBA-down and DBA-up to the rolling windows of EUR/USD are reported in Tables 12 and 13 respectively. If we sum up the profits in Tables 12 and 13, we note that DBA-down and DBA-up generates a total profits of $12.66 \%$ and $25.62 \%$ respectively. Note that if we apply the buyand-hold approach to the applied windows of EUR/USD, it would generates a loss of $-9.2 \%$. We consider these results as an endorsement of the profitability of our trading strategy. In this experiment, DBA-down generates 1919 trades; of which 1233 are winning trades (profit factor is 1.80). Whereas, DBAup generates 2142 trades; of which 1410 are winning trades (profit factor is 1.93).

\section{CONCLUSION}

Unlike time series, which sample prices at fixed intervals, Directional Change (DC) samples prices based on the magnitude, named $\theta$, of price changes. DC segments the market into alternating downtrends and uptrends.

The majority of existing trading strategies use time series. Only few trading models were developed under the DC framework. In this paper, we provide evidences that the DC concept is helpful to develop a consistently profitable trading strategy. We introduce a new contrarian trading strategy, named Backlash Algorithm, or BA for short, which is based on the DC concept. We describe two types of BA: BA-down and BA-up. For each of BA-down and BA-up we provide two versions: static and dynamic. The static versions, named SBA-down and SBA-up, include parameters to be regulated by the trader. Such task may not be easy. The advantage of the dynamic versions of BA, named DBA-down and DBA-up, is that they compute automatically the values of the used parameters.

We provide a set of experiments using two currency pairs, namely EUR/CHF and EUR/USD. We use a rolling window approach to evaluate the performance of BA-down and BA-up. We measure the total profits, profit factor, max drawdown and profitability percent. The experimental results suggest that BA is consistently profitable. These results also show that BAdown and BA-up may have different performance during the same trading period. The results suggest that the dynamic versions perform better than the static versions in most cases. We provide evidence that the performance of BA is affected by the value of $\theta$.

The results of applying Dynamic BA to EUR/CHF show that: 1) the generated total profit range between $59 \%$ and $145 \%$ within seven months; whereas the buy-and-hold approach incurred a loss of $-14 \%$ during the same trading period, and 2) the profit factor ranged between 2.40 and 2.74. We consider the results obtained in this paper as a proof of the effectiveness of our proposed trading strategy; which highlights the usefulness of the DC approach as the cornerstone of Backlash Algorithm.

\section{REFERENCES}

[1] X. Li, Z. Deng and J. Luo, "Trading strategy design in financial investment through a turning points," expert system with application, vol. 36, pp. 7818-7826, 2009.

[2] A. Skabar, "Direction-of-Change Financial Time Series Forecasting using Bayesian Learning for MLPs," in Proceedings of the World Congress on Engineering, London, UK., 2008. pp. 1160-1165.

[3] B. J. Vanstone, T. Hahn and G. Finnie, "Developing HighFrequency Foreign Exchange Trading Systems," in 25th Australasian Finance and Banking Conference, Sydney, 2012. SSRN: http://ssrn.com/abstract=2132390.

[4] A. Esfahanipour and S. Mousavi, " A genetic programming model to generate risk-adjusted technical trading rules in stock markets.," Expert system with application, vol. 38, pp. 84388445, 2011.

[5] M. Shintani, T. Yabub and D. Nagakurac, "Spurious regressions in technical trading.," Journal of Econometrics, vol. 169, pp. 301309, 2012

[6] R. Almgren and J. Lorenz, "Bayesian Adaptive Trading with a Daily Cycle," The Journal of Trading, vol. 1, no. 4, pp. 38-46, 2006.

[7] C. R. M. Daryl, L. K. J. Shawn and C. H. Y. Sabrina, "Profit and Risk-Profit Ratio Based Momentum Strategies: A Fresh Perspective," Journal of Finance and Investment Analysis, vol. 2, no. 1, pp. 1-13, 2013.

[8] J.-S. Chen, J.-L. Hou, S.-M. Wua and Y.-W. Chang-Chien, "Constructing investment strategy portfolios by combination genetic algorithms.," Expert system with application, vol. 36, pp. 3824-3828, 2009.

[9] D. Guillaume, M. Dacorogna, R. Davé, U. Müller, R. Olsen and O. Pictet, "From the bird's eye to the microscope: A survey of new stylized facts of the intra-daily foreign exchange markets," Finance and stochastic, vol. 1, no. 2, pp. 95-129, 1997.

[10] E.P.K. Tsang, R. Tao and S. Ma, Profiling Financial Market Dynamics under Directional Changes, Working Paper WP07415, Centre for Computational Finance and Economic Agents (CCFEA), University of Essex, 2015.

[11] J. Glattfelder, A. Dupuis and R. Olsen, "Patterns in highfrequency FX data: Discovery of 12 empirical scaling laws," Quantitative Finance, vol. 11, no. 4, pp. 599-614, 2011.

[12] T. Bisig, A. Duouis, and V. Impagliazzo, "The scale of market quick," Quantitative Finance, vol. 12, no. 4, pp. 501-508, 2012.

[13] S. Masry, "Event-Based Microscopic Analysis of the FX Market," $\mathrm{PhD}$ thesis, Centre for Computational Finance and Economic Agents (CCFEA). Colchester: University of Essex. 2013.

[14] A. Dupuis and R. Olsen, "High Frequency Finance: Using Scaling Laws to Build Trading Models," in Handbook of Exchange Rates, in: J. James et al, Ed., Wiley, 2012, pp. 563-582.

[15] J. Gypteau, F. Otero and M. Kampouridis, "Directional Changes based trading strategy with genetci programming," in: A. M. Mora, and G.Squillero, Ed., EvoApplications, EvoStar. Springer, 2015, pp. 1-12.

[16] R. Pardo, The Evaluation and Optimization of Trading Strategies, $2^{\text {nd }}$ edition, NJ, USA: John Wiley \& Sons, 2008.

[17] F. Sortino and R. van der Meer, "Downside risk," Journal of Portfolio Management, vol. 17, no. 4, pp. 27-31, 1991. 